\title{
Long-time asymptotics of Hele-Shaw flow for perturbed balls with injection and suction
}

\author{
E. VONDENHOFF ${ }^{\dagger}$ \\ Department of Mathematics and Computer Science, Technische Universiteit Eindhoven, \\ P.O. Box 513, 5600 MB Eindhoven, The Netherlands
}

[Received 12 March 2007 and in revised form 8 July 2008]

\begin{abstract}
We discuss long-time behaviour of Hele-Shaw flow with injection and suction for domains that are small perturbations of balls. An evolution equation for the motion of these domains is derived and linearised. We use spectral properties of the linearisation to show that in the case of injection, perturbations of balls decay algebraically. For classical Hele-Shaw flow, convergence turns out to be faster if low Richardson moments vanish. If for the three-dimensional case surface tension is included, all liquid can be removed by suction if the suction point and the geometric centre coincide and the ratio of suction speed and surface tension is small enough. An arbitrarily large portion of the liquid can be removed if the initial domain is sufficiently close to a ball. The main tools are the principle of linearised stability and H. Amann's theory of abstract quasilinear parabolic evolution equations.
\end{abstract}

2000 Mathematics Subject Classification: 35R35, 35K55, 76D27.

Keywords: Hele-Shaw flow; linearised stability; abstract quasilinear parabolic evolution equations.

\section{Introduction}

In the problem of Hele-Shaw flow with injection or suction at a single point one seeks both a family of moving domains $t \mapsto \Omega(t) \subseteq \mathbb{R}^{N}, 0 \in \Omega(t)$, parameterised by time $t$, and two functions $v(\cdot, t): \Omega(t) \rightarrow \mathbb{R}^{N}$ and $p(\cdot, t): \Omega(t) \rightarrow \mathbb{R}$ such that

$$
\begin{aligned}
\operatorname{div} v & =\mu \delta & & \text { in } \Omega(t), \\
v & =-\nabla p & & \text { in } \Omega(t) .
\end{aligned}
$$

The family $t \mapsto \Omega(t)$ models a liquid domain that moves under influence of injection or suction at the origin. Here $v$ and $p$ are the velocity and pressure field, respectively, $\mu$ stands for the injection speed if $\mu>0$, or the suction speed if $\mu<0$, and $\delta$ is the delta distribution. The evolution of the boundary $\Gamma(t)$ is specified by the requirement that its normal velocity $v_{n}$ is given by

$$
v_{n}=v \cdot n \text {. }
$$

To get a well-defined free boundary problem, a boundary condition has to be added. Here we shall discuss two possibilities.

- Classical Hele-Shaw flow, where

$$
p=0 \quad \text { on } \Gamma(t) .
$$

${ }^{\dagger}$ E-mail: e.vondenhoff@tue.nl 
It is well known that for this problem only the injection case $\mu>0$ is well-posed. By an appropriate scaling of time we can assume $\mu=1$.

- Hele-Shaw flow with surface tension, where

$$
p=-\gamma \kappa \quad \text { on } \Gamma(t)
$$

Here, $\kappa(\cdot, t): \Gamma(t) \rightarrow \mathbb{R}$ stands for the mean curvature of the moving boundary $t \mapsto \Gamma(t)$ (taken negative if $\Omega(t)$ is convex) and $\gamma$ is a positive constant. For this problem both the injection and the suction case will be studied.

Equations (1.1) and (1.2) give

$$
\Delta p=-\mu \delta .
$$

Together with (1.4) or 1.5, this forms a Dirichlet problem for any time $t$. On $\Gamma(t)$ we have, by 1.2) and 1.3,

$$
v_{n}=-\frac{\partial p}{\partial n}
$$

Besides liquid flow in a Hele-Shaw cell [8], the model and variations of it describe the growth of tumors [5] and porous media flow [10, 11].

For similar problems, Escher and Simonett [10, 13, 12] proved existence of short-time solutions $t \mapsto \Omega(t)$. For the problem [1.1]-1.4) weak solutions have been investigated by Gustafsson [16] and Begehr and Gilbert [4]. For the suction problem for (1.1)-(1.3), [1.5], Tian [23] proved that if the geometric centre and the suction point do not coincide, then the solution breaks down before all liquid is sucked out or the domain becomes unbounded with zero area. Prokert [22] proved short-time existence and uniqueness of solutions for the problem with $\gamma>0$ and $\mu=0$. Global existence and exponential decay are proved for this problem in the same paper and in [15] for the case where the initial domain is sufficiently close to a ball. The main tools are linearisation around the equilibrium and energy estimates. In [14] a similar result has been proved for the two-phase problem. In [6] an evolution equation for a conformal mapping in the complex plane is derived to prove a corresponding result for the exterior problem in two dimensions.

Let $\sigma_{N}$ be the area of the unit sphere $\mathbb{S}^{N-1}$ in $\mathbb{R}^{N}$. We will assume that the initial domain $\Omega(0)$ has the same volume as the unit ball $\mathbb{B}^{N}$ in $\mathbb{R}^{N}$, i.e. $|\Omega(0)|=\sigma_{N} / N$. The volume $V(t)$ of the domain satisfies

$$
V(t)=\frac{\sigma_{N}}{N}+\mu t
$$

because of

$$
\frac{\mathrm{d} V}{\mathrm{~d} t}=\frac{\mathrm{d}}{\mathrm{d} t} \int_{\Omega(t)} \mathrm{d} x=\int_{\Gamma(t)} v_{n} \mathrm{~d} \sigma=\int_{\Omega(t)} \operatorname{div} v \mathrm{~d} x=\mu .
$$

Note that for negative $\mu$, our problem can only make sense if

$$
t \leqslant T_{\mu}:=-\frac{\sigma_{N}}{\mu N}
$$

By radial symmetry, if $\Omega(0)=\mathbb{B}^{N}$ then $\Omega(t)=s_{N, \mu}(t) \mathbb{B}^{N}$, where

$$
s_{N, \mu}(t)=\sqrt[N]{\frac{\mu N t}{\sigma_{N}}+1} .
$$


In order to prove stability of these solutions, we rescale by a factor $s_{N, \mu}(t)$ such that $\mathbb{B}^{N}$ becomes a stationary solution. Star-shaped perturbations of this stationary solution are described by means of a small function $r(\cdot, t): \mathbb{S}^{N-1} \rightarrow \mathbb{R}$.

This paper is organised as follows. In Section 2 we derive a nonlinear nonlocal parabolic evolution equation for $r(t)$. For the classical Hele-Shaw problem the evolution operator can be treated as autonomous after introducing a new time variable. The linearisation of the evolution operator around the stationary solution is of first order and essentially given by the Dirichlet-toNeumann operator for the Laplacian on $\mathbb{B}^{N}$. For the problem with surface tension, only the case $N=3$ can be treated as autonomous. The linearisation is a third order polynomial in the Dirichletto-Neumann operator.

The spectral properties of the linearised operator for classical Hele-Shaw flow in little Hölder spaces are discussed in Section 3. Based on the spectrum, a global existence result is proved and it is shown that $r(t)$ decays algebraically. Here we use the principle of linearised stability (see [19]). In Section 4 we show that convergence is faster for domains for which low Richardson moments vanish. This is done by discussing the linearisation of the evolution operator restricted to the corresponding invariant manifolds.

From Section 5 on we restrict our attention to the problem with surface tension in $\mathbb{R}^{3}$. For the case of injection, we prove existence of small solutions for all $t>0$. In the case of suction, we find that all liquid can be removed under the conditions that suction takes place in the geometric centre of the liquid domain and the ratio $|\mu| / \gamma$ is small enough. This gives a partial answer to a problem posed in 1993 [17]. Furthermore, we get decay properties that show that the liquid domain vanishes "as a round point". In Section 6 we prove the following stability result: an arbitrarily large portion of liquid can be removed if the initial domain is close enough to a ball. There we use the fact that the evolution induces a semiflow on appropriate function spaces.

In future research we intend to discuss the problem with surface tension for $N \neq 3$. The problem is not autonomous and therefore the principle of linearised stability cannot be used. We plan to study long-time asymptotics of Stokes flow with injection and suction.

\section{The evolution equation for the domain}

In this section we derive a nonlinear nonlocal evolution equation describing the motion of the domain, in a rescaled version. Furthermore, we determine the linearisation of the evolution operator in terms of the Dirichlet-to-Neumann mapping.

Consider moving domains $\Omega(t)$ that can be described by a continuous function $R: \mathbb{S}^{N-1} \times$ $[0, \infty) \rightarrow(-1, \infty)$ such that

$$
\Omega(t)=\Omega_{R(\cdot, t)}:=\left\{x \in \mathbb{R}^{N} \backslash\{0\}:|x|<1+R(x /|x|, t)\right\} \cup\{0\} .
$$

Sometimes we will write $R(t)$ instead of $R(\cdot, t)$. Define

$$
r(t)=\frac{1+R(t)}{s_{N, \mu}(t)}-1 .
$$

From this we get

$$
\Omega_{r(t)}=s_{N, \mu}(t)^{-1} \Omega_{R(t)} .
$$

We derive an evolution equation for perturbations $r$ of the stationary solution $r(t) \equiv 0$, and investigate long-time behaviour. 
Now we shall consider the problem for fixed time and suppress the argument $t$. Define

$$
\Gamma_{R}:=\partial \Omega_{R}=\left\{(1+R(\xi)) \xi: \xi \in \mathbb{S}^{N-1}\right\}
$$

Let $\tilde{z}(R)=\tilde{z}(R, \cdot): \mathbb{S}^{N-1} \rightarrow \Gamma_{R}$ be the mapping given by

$$
\tilde{z}(R, \xi)=(1+R(\xi)) \xi .
$$

The free boundary problem $1.1-(1.4)$ or $1.1-1.3,1.5)$ respectively translates into the following evolution equation for $R$ :

$$
\frac{\partial R}{\partial t}(\xi)=-\frac{\nabla p(\tilde{z}(R, \xi)) \cdot n(R, \xi)}{n(R, \xi) \cdot \xi}, \quad \xi \in \mathbb{S}^{N-1},
$$

where $n(R)=n(R, \cdot)$ maps an element $\xi \in \mathbb{S}^{N-1}$ to the exterior unit normal vector on $\Gamma_{R}$ at the point $\tilde{z}(R, \xi)$. For this we refer to [21, Chapter 3]. We shall write this evolution equation in terms of $r$.

Define $\Phi: \mathbb{R}^{N} \rightarrow \mathbb{R}$ by

$$
\Phi(x):= \begin{cases}-\frac{1}{2 \pi} \ln |x|, & N=2, \\ \frac{1}{(N-2) \sigma_{N}|x|^{N-2}}-\frac{1}{(N-2) \sigma_{N}}, & N \geqslant 3,\end{cases}
$$

and $U: \Omega_{R} \rightarrow \mathbb{R}$ by

$$
U=p-\mu \Phi
$$

Because $\Delta \Phi=-\delta$ we have

$$
\begin{aligned}
& \Delta U=0 \quad \text { in } \Omega(t), \\
& U=-\gamma \kappa_{R}-\mu \Phi \quad \text { on } \Gamma(t) \text {. }
\end{aligned}
$$

Here $\kappa_{R}: \Gamma_{R} \rightarrow \mathbb{R}$ stands for the mean curvature of $\Gamma_{R}$. From

$$
\nabla \Phi(x)=-\frac{1}{\sigma_{N}|x|^{N}} x
$$

2.1 and 2.2 we get

$$
\frac{\partial R}{\partial t}(\xi)=-\frac{\nabla U(\tilde{z}(R, \xi)) \cdot n(r, \xi)}{n(r, \xi) \cdot \xi}+\frac{\mu}{\sigma_{N} s_{N, \mu}^{N-1}(1+r(\xi))^{N-1}},
$$

since $r(\xi)>-1$ and $n(R, \xi)=n(r, \xi)$. Define $u: \Omega_{r} \rightarrow \mathbb{R}$ by

$$
u(x)=U\left(s_{N, \mu} x\right) .
$$

Then $\Delta u=0$ and on $\Gamma_{r}$,

$$
u(x)=-\gamma \kappa_{R}\left(s_{N, \mu} x\right)-\mu \Phi\left(s_{N, \mu} x\right)=-\gamma s_{N, \mu}^{-1} \kappa_{r}(x)-\mu s_{N, \mu}^{2-N} \Phi(x)+\mu C_{N},
$$


for some function $C_{N}$ depending on $t$. Let $\Lambda_{r}: \Omega_{r} \rightarrow \mathbb{R}$ and $G_{r}: \Omega_{r} \rightarrow \mathbb{R}$ be the harmonic functions that satisfy

$$
\Lambda_{r}=\Phi \quad \text { on } \Gamma_{r}, \quad G_{r}=\kappa_{r} \quad \text { on } \Gamma_{r} .
$$

Then

$$
u=-\gamma s_{N, \mu}^{-1} G_{r}-\mu s_{N, \mu}^{2-N} \Lambda_{r}+\mu C_{N}
$$

We have

$$
\nabla U(\tilde{z}(R))=s_{N, \mu}^{-1} \nabla u(\tilde{z}(r))=-\gamma s_{N, \mu}^{-2} \nabla G_{r}(\tilde{z}(r))-\mu s_{N, \mu}^{1-N} \nabla \Lambda_{r}(\tilde{z}(r))
$$

Using

we find from (2.4) that

$$
s_{N, \mu}^{\prime}=\frac{\mu}{\sigma_{N}} s_{N, \mu}^{1-N}
$$

$$
\begin{aligned}
\frac{\partial r}{\partial t}(\xi)= & \frac{\gamma}{s_{N, \mu}(t)^{3}} \frac{\nabla G_{r}(\tilde{z}(r, \xi)) \cdot n(r, \xi)}{n(r, \xi) \cdot \xi} \\
& +\frac{\mu}{s_{N, \mu}(t)^{N}}\left(\frac{\nabla \Lambda_{r}(\tilde{z}(r, \xi)) \cdot n(r, \xi)}{n(r, \xi) \cdot \xi}+\frac{1}{\sigma_{N}(1+r(\xi))^{N-1}}-\frac{1+r(\xi)}{\sigma_{N}}\right) .
\end{aligned}
$$

In order to transform our free boundary problems to the fixed reference domain $\overline{\mathbb{B}^{N}}$, the righthand side has to be written in terms of operators on function spaces on $\overline{\mathbb{B}^{N}}$ and $\mathbb{S}^{N-1}$. For $k \in \mathbb{N}_{0}$ and $\alpha \in(0,1)$, let the little Hölder spaces $h^{k, \alpha}(K)$ on a compact domain $K$ be defined as the closure of $\mathcal{C}^{\infty}(K)$ in the Hölder spaces $\mathcal{C}^{k, \alpha}(K)$. These spaces have the property that $h^{k, \alpha}(K)$ is dense in $h^{k^{\prime}, \alpha^{\prime}}(K)$ if $k^{\prime}+\alpha^{\prime}<k+\alpha$.

By [19, Theorem 0.3.2] there exists an extension operator $E \in \mathcal{L}\left(\mathcal{C}^{k, \alpha}\left(\mathbb{S}^{N-1}\right), \mathcal{C}^{k, \alpha}\left(\overline{\mathbb{B}^{N}}\right)\right)$ for $k \in\{0,1,2\}$ and $\alpha \in[0,1)$, with the property

$$
\left.E(r)\right|_{\mathbb{S}^{N-1}}=r .
$$

Introduce the mapping $z: \mathcal{C}^{2, \alpha}\left(\mathbb{S}^{N-1}\right) \rightarrow\left(\mathcal{C}^{2, \alpha}\left(\overline{\mathbb{B}^{N}}\right)\right)^{N}$ as

$$
z(r, x)=(1+E(r, x)) x,
$$

where $z(r, \cdot)=z(r)$ and $E(r, \cdot)=E(r)$.

LEMma 2.1 There exists a $\delta>0$ such that if $\|r\|_{\mathcal{C}^{2, \alpha}\left(\mathbb{S}^{N-1}\right)}<\delta$ then $z(r): \overline{\mathbb{B}^{N}} \rightarrow \bar{\Omega}_{r}$ is bijective.

Proof. This follows from the mean value theorem. A complete proof is given in [25, Lemma 2.2].

Define $\mathcal{J}: \mathcal{C}^{2, \alpha}\left(\mathbb{S}^{N-1}\right) \rightarrow\left(\mathcal{C}^{1, \alpha}\left(\overline{\mathbb{B}^{N}}\right)\right)^{N \times N}$ by

$$
\mathcal{J}(r)=\frac{\partial z(r)}{\partial x}
$$

Again $\mathcal{J}(r, \cdot)=\mathcal{J}(r)$.

Lemma 2.2 There exists a $\delta>0$ such that if $r \in \mathcal{C}^{2, \alpha}\left(\mathbb{S}^{N-1}\right)$ satisfies $\|r\|_{\mathcal{C}^{2, \alpha}\left(\mathbb{S}^{N-1}\right)}<\delta$, then $\mathcal{J}(r, x)$ is an invertible matrix for every $x \in \overline{\mathbb{B}^{N}}$ and $x \mapsto \mathcal{J}(r, x)^{-1} \in\left(\mathcal{C}^{1, \alpha}\left(\overline{\mathbb{B}^{N}}\right)\right)^{N \times N}$. Furthermore, $z(r)^{-1} \in\left(\mathcal{C}^{2, \alpha}\left(\bar{\Omega}_{r}\right)\right)^{N}$. 
Proof. This follows from the fact that the Hölder spaces are Banach algebras. For more detail we refer to [25, Lemma 2.3].

We denote the components of $\mathcal{J}(r)^{-1}$ by $j^{i, k}(r)$. By Lemmas 2.1 and 2.2 we see that there exists a neighbourhood $\mathcal{U}$ of 0 in $\mathcal{C}^{2, \alpha}\left(\mathbb{S}^{N-1}\right)$ and two mappings $\mathcal{A}: \mathcal{U} \rightarrow \mathcal{L}\left(\mathcal{C}^{2, \alpha}\left(\overline{\mathbb{B}^{N}}\right), \mathcal{C}^{0, \alpha}\left(\overline{\mathbb{B}^{N}}\right)\right)$ and $\mathcal{Q}: \mathcal{U} \rightarrow \mathcal{L}\left(\mathcal{C}^{2, \alpha}\left(\overline{\mathbb{B}^{N}}\right),\left(\mathcal{C}^{1, \alpha}\left(\overline{\mathbb{B}^{N}}\right)\right)^{N}\right)$ such that

$$
\mathcal{A}(r) u=\left(\Delta\left(u \circ z(r)^{-1}\right)\right) \circ z(r)=\sum_{i, k, l} j^{i, l}(r) \frac{\partial}{\partial x_{i}}\left(j^{k, l}(r) \frac{\partial u}{\partial x_{k}}\right)
$$

and

$$
\mathcal{Q}(r) u=\left(\nabla\left(u \circ z(r)^{-1}\right)\right) \circ z(r)=\sum_{i, k} j^{k, i}(r) \frac{\partial u}{\partial x_{k}} e_{i},
$$

where $e_{i}$ is the $i$-th unit vector in $\mathbb{R}^{N}$. Let $\mathcal{P}: \mathcal{U} \rightarrow \mathcal{L}\left(\mathcal{C}^{2, \alpha}\left(\overline{\mathbb{B}^{N}}\right), \mathcal{C}^{0, \alpha}\left(\overline{\mathbb{B}^{N}}\right) \times \mathcal{C}^{2, \alpha}\left(\mathbb{S}^{N-1}\right)\right)$ be defined by

$$
\mathcal{P}(r) u=\left(\begin{array}{c}
\mathcal{A}(r) u \\
\operatorname{Tr} u
\end{array}\right)
$$

Because $\mathcal{P}(0)$ is invertible, $\mathcal{P}(r)$ is invertible as well for small $r$. Let $\phi: \mathcal{U} \rightarrow \mathcal{C}^{2, \alpha}\left(\mathbb{S}^{N-1}\right)$ be

$$
\phi(r, x)=\Phi((1+r(x)) x),
$$

where $\phi(r, \cdot)=\phi(r)$ and $\Phi: \mathbb{R}^{N} \rightarrow \mathbb{R}$ is defined by 2.3. Introduce $\kappa(r)$ as the function that maps an element $\xi \in \mathbb{S}^{N-1}$ to the curvature of $\Gamma_{r}$ at $z(r, \xi)$.

We can choose $\mathcal{U}$ such that we can define $\mathcal{E}: \mathcal{U} \rightarrow \mathcal{L}\left(\mathcal{C}^{2, \alpha}\left(\mathbb{S}^{N-1}\right), \mathcal{C}^{1, \alpha}\left(\mathbb{S}^{N-1}\right)\right)$ and $l: \mathcal{U} \rightarrow$ $\mathcal{C}^{2, \alpha}\left(\mathbb{S}^{N-1}\right)$ by

$$
(\mathcal{E}(r) \psi)(\xi)=-\frac{\operatorname{Tr}\left(\mathcal{Q}(r)\left[\mathcal{P}(r)^{-1}\left[\begin{array}{l}
0 \\
\psi
\end{array}\right]\right]\right)(\xi) \cdot n(r, \xi)}{n(r, \xi) \cdot \xi}
$$

and

$$
l(r)=\frac{1}{\sigma_{N}(1+r)^{N-1}}-\frac{1+r}{\sigma_{N}} .
$$

Now 2.5) gets the form

$$
\frac{\partial r}{\partial t}=\frac{\gamma}{s_{N, \mu}(t)^{3}} \mathcal{F}_{1}(r)+\frac{\mu}{s_{N, \mu}(t)^{N}} \mathcal{F}_{2}(r)
$$

where

$$
\mathcal{F}_{1}(r)=-\mathcal{E}(r) \kappa(r) \quad \text { and } \quad \mathcal{F}_{2}(r)=-\mathcal{E}(r) \phi(r)+l(r) .
$$

Introduce a new time variable $\tau=\tau(t)$ such that $\tau(0)=0$ and

$$
\frac{d \tau}{d t}=\frac{1}{s_{N, \mu}(t)^{N}}=\frac{1}{\frac{\mu N t}{\sigma_{N}}+1}
$$

thus

$$
\tau(t)=\frac{\sigma_{N}}{\mu N} \ln \left(\frac{\mu N t}{\sigma_{N}}+1\right)
$$


- For the classical Hele-Shaw flow $(\gamma=0, \mu=1)$ we get an autonomous evolution equation

$$
\frac{\partial \bar{r}}{\partial \tau}=\mathcal{F}_{2}(\bar{r}), \quad \text { where } \quad \bar{r}(\tau)=r(t) .
$$

- For the problem with surface tension, only the equation for $N=3$ becomes autonomous. In this case we get

$$
\frac{\partial \bar{r}}{\partial \tau}=\mathcal{F}_{\gamma, \mu}(\bar{r}):=\gamma \mathcal{F}_{1}(\bar{r})+\mu \mathcal{F}_{2}(\bar{r}) .
$$

In the following we will write $r$ instead of $\bar{r}$.

LEMma 2.3 (i) There exists a neighbourhood $\mathcal{U}$ of zero in $h^{2, \alpha}\left(\mathbb{S}^{N-1}\right)$ such that the operator $\mathcal{E}: \mathcal{U} \rightarrow \mathcal{L}\left(\mathcal{C}^{2, \alpha}\left(\mathbb{S}^{N-1}\right), \mathcal{C}^{1, \alpha}\left(\mathbb{S}^{N-1}\right)\right)$ is analytic.

(ii) There exists a neighbourhood $\mathcal{U}_{1}$ of zero in $h^{4, \alpha}\left(\mathbb{S}^{N-1}\right)$ such that the operator $\mathcal{F}_{1}: \mathcal{U}_{1} \rightarrow$ $h^{1, \alpha}\left(\mathbb{S}^{N-1}\right)$ is analytic.

(iii) There exists a neighbourhood $\mathcal{U}_{2}$ of zero in $h^{2, \alpha}\left(\mathbb{S}^{N-1}\right)$ such that the operator $\mathcal{F}_{2}: \mathcal{U}_{2} \rightarrow$ $h^{1, \alpha}\left(\mathbb{S}^{N-1}\right)$ is analytic.

Proof. This can be obtained in the same way as was done in [21, Chapter 3] for Sobolev spaces. The proof is based on the fact that all components of $\mathcal{E}$ can be expressed in terms of $z$, the fact that compositions and inversion of analytic operators in Banach algebras are analytic, and the implicit function theorem. For more details we refer to [25, Chapter 2] and [24, Lemma 2.3].

The next step is finding the linearisation of the evolution operators around zero.

Lemma 2.4 The Fréchet derivative of $\mathcal{F}_{2}$ at 0 satisfies

$$
\mathcal{F}_{2}^{\prime}(0)[h]=-\frac{1}{\sigma_{N}} \mathcal{N} h-\frac{N}{\sigma_{N}} h,
$$

where $\mathcal{N}: \mathcal{C}^{2, \alpha}\left(\mathbb{S}^{N-1}\right) \rightarrow \mathcal{C}^{1, \alpha}\left(\mathbb{S}^{N-1}\right)$ is the Dirichlet-to-Neumann operator on the unit ball given by

$$
\mathcal{N} h=\operatorname{Tr} \nabla \mathcal{P}(0)^{-1}\left(\begin{array}{l}
0 \\
h
\end{array}\right) \cdot n(0) .
$$

Proof. Let $\Pi: \mathcal{U} \rightarrow \mathcal{C}^{2, \alpha}\left(\overline{\mathbb{B}^{N}}\right)$ be defined by

$$
\Pi(r)=\mathcal{P}(r)^{-1}\left(\begin{array}{c}
0 \\
-\phi(r)
\end{array}\right) .
$$

From $\mathcal{A}(r) \Pi(r)=0, \Pi(0)=0$ and $\phi^{\prime}(0)[h]=-\frac{1}{\sigma_{N}} h$ we get

$$
\Pi^{\prime}(0)[h]=\frac{1}{\sigma_{N}} \mathcal{P}(0)^{-1}\left(\begin{array}{l}
0 \\
h
\end{array}\right) .
$$

We can write $\mathcal{F}_{2}(r)$ as

$$
\mathcal{F}_{2}(r)=-\frac{\mathcal{Q}(r) \Pi(r) \cdot n(r)}{n(r) \cdot \mathcal{I}}+\frac{1}{\sigma_{N}(1+r)^{N-1}}-\frac{1+r}{\sigma_{N}} .
$$


From a Taylor expansion we get

$$
\mathcal{F}_{2}^{\prime}(0)[h]=-\frac{\operatorname{Tr} \mathcal{Q}(0) \Pi^{\prime}(0)[h] \cdot n(0)}{n(0) \cdot \mathcal{I}}-\frac{N}{\sigma_{N}} h=-\operatorname{Tr} \nabla \Pi^{\prime}(0)[h] \cdot n(0)-\frac{N}{\sigma_{N}} h,
$$

where $\mathcal{I}$ is the identity.

LEMMA 2.5 The Fréchet derivative of $\mathcal{F}_{1}$ at 0 satisfies

$$
\mathcal{F}_{1}^{\prime}(0)[h]=\mathcal{N}\left(\kappa^{\prime}(0)[h]\right)=\mathcal{N}\left(\Delta_{0} r+(N-1) r\right)=\mathcal{N}\left(-\mathcal{N}^{2} r-(N-2) \mathcal{N} r+(N-1) r\right) .
$$

Proof. From [21, Chapter 6] we have

$$
\kappa^{\prime}(0)[h]=\Delta_{0} h+(N-1) h,
$$

where $\Delta_{0}$ denotes the Laplace-Beltrami operator on the unit sphere. From [20] we have

$$
\Delta_{0} r=-\mathcal{N}^{2} r-(N-2) \mathcal{N} r
$$

The proof can be completed as for Lemma 2.4

\section{The spectrum of the linearisation and stability for the nonlinear autonomous evolution in the case of classical Hele-Shaw flow}

In this section we apply the principle of linearised stability to the evolution equation 2.14 for the classical Hele-Shaw problem, in order to derive a stability result for the stationary solution $r \equiv 0$. For this purpose we study the spectral properties of the operator $\mathcal{F}_{2}^{\prime}(0): h^{2, \alpha}\left(\mathbb{S}^{N-1}\right) \rightarrow$ $h^{1, \alpha}\left(\mathbb{S}^{N-1}\right)$ given by 2.16 . First we find the eigenvalues and eigenfunctions of the Dirichlet-toNeumann operator $\mathcal{N}: h^{2, \alpha}\left(\mathbb{S}^{N-1}\right) \rightarrow h^{1, \alpha}\left(\mathbb{S}^{N-1}\right)$.

We start by defining $H_{k}^{N}$ as the vector space of harmonic homogeneous polynomials of degree $k$ in $N$ variables. Spherical harmonics are defined as the restriction of these polynomials to the unit sphere,

$$
S_{k}^{N}=\left\{\left.q\right|_{\mathbb{S}^{N-1}}: q \in H_{k}^{N}\right\}
$$

The dimensions $\operatorname{dim} S_{k}^{N}=: v(N, k)$ are finite. In particular, $v(2, k)=2$ for $k \neq 0, v(2,0)=1$ and $v(3, k)=2 k+1$. For each $S_{k}^{N}$ we can choose an orthonormal basis with respect to the $\mathbb{L}_{2}\left(\mathbb{S}^{N-1}\right)$ inner product,

$$
S_{k}^{N}=\left\langle s_{k, 1}^{N}, \ldots, s_{k, v(N, k)}^{N}\right\rangle .
$$

We shall often suppress the index $N$ in $s_{k, j}^{N}$. It is well known that

$$
\mathcal{N} s=k s
$$

for $s \in S_{k}^{N}$ ([20, Lemma 1]). The spherical harmonics

$$
\bigcup_{k=0}^{\infty}\left\{s_{k, 1}, \ldots, s_{k, v(N, k)}\right\}
$$

form an orthonormal basis for $\mathbb{L}_{2}\left(\mathbb{S}^{N-1}\right)$. Therefore, from 2.16 we get the following result. 
COROLLARY 3.1 The set of eigenvalues of $\mathcal{N}: h^{2, \alpha}\left(\mathbb{S}^{N-1}\right) \rightarrow h^{1, \alpha}\left(\mathbb{S}^{N-1}\right)$ is $\mathbb{N}_{0}$. The eigenspace corresponding to the eigenvalue $k \in \mathbb{N}_{0}$ is $S_{k}^{N}$. The point spectrum of $\mathcal{F}_{2}^{\prime}(0)$ is therefore

$$
\pi\left(\mathcal{F}_{2}^{\prime}(0)\right)=\left\{-\frac{N}{\sigma_{N}},-\frac{N+1}{\sigma_{N}},-\frac{N+2}{\sigma_{N}}, \ldots\right\}
$$

and the eigenspace for the eigenvalue $-(N+k) / \sigma_{N}$ is $S_{k}^{N}$.

Now we will prove that all elements of the spectrum of $\mathcal{F}_{2}^{\prime}(0): h^{2, \alpha}\left(\mathbb{S}^{N-1}\right) \rightarrow h^{1, \alpha}\left(\mathbb{S}^{N-1}\right)$ are eigenvalues, by showing that the resolvent $\left(\lambda \mathcal{I}-\mathcal{F}_{2}^{\prime}(0)\right)^{-1}: h^{1, \alpha}\left(\mathbb{S}^{N-1}\right) \rightarrow h^{1, \alpha}\left(\mathbb{S}^{N-1}\right)$ is compact for suitable $\lambda \in \mathbb{C}$.

LEMMA 3.2 The spectrum of $\mathcal{F}_{2}^{\prime}(0): h^{2, \alpha}\left(\mathbb{S}^{N-1}\right) \rightarrow h^{1, \alpha}\left(\mathbb{S}^{N-1}\right)$ consists entirely of eigenvalues:

$$
\sigma\left(\mathcal{F}_{2}^{\prime}(0)\right)=\pi\left(\mathcal{F}_{2}^{\prime}(0)\right)=\left\{-\frac{N}{\sigma_{N}},-\frac{N+1}{\sigma_{N}},-\frac{N+2}{\sigma_{N}}, \ldots\right\} .
$$

Proof. By [9, Appendix B, Theorems B.3 and B.4] we see that $\mathcal{F}_{2}^{\prime}(0)$ generates an analytic semigroup on $h^{1, \alpha}\left(\mathbb{S}^{N-1}\right)$ with dense domain of definition $h^{2, \alpha}\left(\mathbb{S}^{N-1}\right)$. This implies that the resolvent set of $\mathcal{F}_{2}^{\prime}(0)$ is not empty. From the compactness of the embedding $h^{2, \alpha}\left(\mathbb{S}^{N-1}\right) \hookrightarrow$ $h^{1, \alpha}\left(\mathbb{S}^{N-1}\right)([1$, Theorem 8.6]) we get existence of a $\lambda \in \mathbb{C}$ such that

$$
\left(\lambda \mathcal{I}-\mathcal{F}_{2}^{\prime}(0)\right)^{-1}: h^{1, \alpha}\left(\mathbb{S}^{N-1}\right) \rightarrow h^{1, \alpha}\left(\mathbb{S}^{N-1}\right)
$$

is compact. From [18, Theorem III.6.29] we get the desired result.

THEOREM 3.3 Let $0<\lambda_{0}<N / \sigma_{N}$. There exists a $\delta>0$ and an $M>0$ such that the problem

$$
\frac{\partial r}{\partial \tau}=\mathcal{F}_{2}(r)
$$

with $r(0)=r_{0} \in h^{2, \alpha}\left(\mathbb{S}^{N-1}\right)$ and $\left\|r_{0}\right\|_{\mathcal{C}^{2, \alpha}\left(\mathbb{S}^{N-1}\right)}<\delta$ has a solution $r \in \mathcal{C}\left([0, \infty), h^{2, \alpha}\left(\mathbb{S}^{N-1}\right)\right) \cap$ $\mathcal{C}^{1}\left([0, \infty), h^{1, \alpha}\left(\mathbb{S}^{N-1}\right)\right)$ satisfying

$$
\|r(\tau)\|_{\mathcal{C}^{2, \alpha}\left(\mathbb{S}^{N-1}\right)} \leqslant M e^{-\lambda_{0} \tau}\left\|r_{0}\right\|_{\mathcal{C}^{2, \alpha}\left(\mathbb{S}^{N-1}\right)} .
$$

Proof. As mentioned before, $\mathcal{F}_{2}^{\prime}(0)$ generates an analytic semigroup on $h^{1, \alpha}\left(\mathbb{S}^{N-1}\right)$ with dense domain of definition $h^{2, \alpha}\left(\mathbb{S}^{N-1}\right)$. Because of Lemma 3.2 the spectrum is left of the imaginary axis and it has distance $-N / \sigma_{N}$ to it. Therefore we can apply [19, Theorem 9.1.2] to show the global existence of $r$ in time and the estimate.

Combining the above with 2.13 we get the following estimate for the nonautonomous problem (2.12):

$$
\|r(t)\|_{\mathcal{C}^{2, \alpha}\left(\mathbb{S}^{N-1}\right)} \leqslant M\left(\frac{N t}{\sigma_{N}}+1\right)^{-\zeta}\left\|r_{0}\right\|_{\mathcal{C}^{2, \alpha}\left(\mathbb{S}^{N-1}\right)}
$$

for $\zeta=\lambda_{0} \sigma_{N} / N$ 


\section{Faster convergence in absence of low-order moments}

In this section we show for classical Hele-Shaw flow that if the integrals of harmonic polynomials with low degrees over the moving domain vanish, then convergence to the equilibrium will be faster.

Let $K \in \mathbb{N}_{0}$ and define

$$
M_{K}^{N}=\left\{r \in h^{2, \alpha}\left(\mathbb{S}^{N-1}\right): \int_{\Omega_{r}} \mathrm{~d} x=\frac{\sigma_{N}}{N} \wedge \int_{\Omega_{r}} h(x) \mathrm{d} x=0 \forall h \in \bigcup_{j=1}^{K} H_{j}^{N}\right\} .
$$

We have $r \in M_{K}^{N}$ if and only if the volume of the corresponding domain $\Omega_{r}$ is equal to the volume of the unit ball and the Richardson moments of order $1, \ldots, K$ vanish.

Lemma 4.1 Let $r$ be a solution of 2.14. If $r(0)$ is in $M_{K}^{N}$ then $r(t) \in M_{K}^{N}$ for all $t \geqslant 0$.

Proof. It follows from our choice of scaling that if the initial domain $\Omega_{r(0)}$ has the same volume as the unit ball, then this will also be the case for $\Omega_{r(t)}$ for all $t>0$. Let $h$ be harmonic, $R(t)=$ $s_{N, \mu}(t)(1+r(t))-1$, and let $p$ and $v$ be the solutions of (1.1p-(1.4) on the corresponding domains $\Omega_{R(t)}$. From Green's identities, (1.3), 11.4, (1.6) and (1.7) it follows that

$$
\frac{\mathrm{d}}{\mathrm{d} t} \int_{\Omega_{R(t)}} h \mathrm{~d} x=\int_{\Gamma_{R(t)}} h v \cdot n \mathrm{~d} x=-\int_{\Gamma_{R(t)}} h \frac{\partial p}{\partial n} \mathrm{~d} x=-\int_{\Gamma_{R(t)}} h \Delta p=h(0) .
$$

This means that integrals of harmonic homogeneous polynomials of nonzero degree over $\Omega_{R(t)}$ are constant in $t$. This completes the proof.

Let $h_{k, j}$ be the harmonic homogeneous polynomials that satisfy

$$
\left.h_{k, j}\right|_{\mathbb{S}^{N-1}}=s_{k, j} .
$$

For $K, L \in \mathbb{N}_{0}$ define the Banach spaces

$$
h_{K}^{L, \alpha}\left(\mathbb{S}^{N-1}\right)=\left\{r \in h^{L, \alpha}\left(\mathbb{S}^{N-1}\right):\left(r, s_{k, j}\right)_{\mathbb{L}_{2}\left(\mathbb{S}^{N-1}\right)}=0, k \leqslant K\right\},
$$

the index set

$$
\mathbb{I}_{K}=\{(k, j): 0 \leqslant k \leqslant K, 1 \leqslant j \leqslant v(N, k)\}
$$

and the map $f_{K}: h^{2, \alpha}\left(\mathbb{S}^{N-1}\right) \rightarrow \mathbb{R}^{\mathbb{I}_{K}}$ by

$$
f_{K}(r)_{k, j}=\int_{\Omega_{r}} h_{k, j} \mathrm{~d} x-\frac{\sqrt{\sigma_{N}}}{N} \delta_{k, 0} .
$$

Note that if we choose $h_{0,0}=1 / \sqrt{\sigma_{N}}$ then $f_{K}(r)=0$ for $r \in M_{K}^{N}$. Let $\mathcal{P}_{K}: h^{2, \alpha}\left(\mathbb{S}^{N-1}\right) \rightarrow$ $h_{K}^{2, \alpha}\left(\mathbb{S}^{N-1}\right)$ be the orthogonal projection onto $h_{K}^{2, \alpha}\left(\mathbb{S}^{N-1}\right)$ with respect to the $\mathbb{L}_{2}$-inner product and define $\phi_{K}: h^{2, \alpha}\left(\mathbb{S}^{N-1}\right) \rightarrow \mathbb{R}^{\mathbb{I}_{K}} \times h_{K}^{2, \alpha}\left(\mathbb{S}^{N-1}\right)$ by

$$
\phi_{K}(r)=\left(\begin{array}{c}
f_{K}(r) \\
\mathcal{P}_{K} r
\end{array}\right) .
$$

Because $h_{k, j}(x)=|x|^{k} s_{k, j}(x /|x|)$ we have

$$
f_{K}(r)_{k, j}=\int_{\mathbb{S}^{N-1}} \frac{(1+r(\varphi))^{k+N}}{k+N} s_{k, j}(\varphi) \mathrm{d} \varphi-\frac{\sqrt{\sigma_{N}}}{N} \delta_{k, 0},
$$


where $\varphi=x /|x| \in \mathbb{S}^{N-1}$. The operators $f_{K}: h^{2, \alpha}\left(\mathbb{S}^{N-1}\right) \rightarrow \mathbb{R}^{\mathbb{I} K}$ are analytic, and Fréchet differentiation leads to

$$
f_{K}^{\prime}(0)[r]_{k, j}=\left(r, s_{k, j}\right)_{\mathbb{L}_{2}\left(\mathbb{S}^{N-1}\right)} .
$$

By linearity of $\mathcal{P}_{K}$ we have

$$
\phi_{K}^{\prime}(0)[r]=\left(\begin{array}{c}
f_{K}^{\prime}(0)[r] \\
\mathcal{P}_{K} r
\end{array}\right)
$$

It is easy to see that $\phi_{K}^{\prime}(0): h^{2, \alpha}\left(\mathbb{S}^{N-1}\right) \rightarrow \mathbb{R}^{\mathbb{I}_{K}} \times h_{K}^{2, \alpha}\left(\mathbb{S}^{N-1}\right)$ is bijective. By the implicit function theorem, $\phi_{K}$ is a diffeomorphism in a neighbourhood of 0 in $h^{2, \alpha}\left(\mathbb{S}^{N-1}\right)$. There exists an open neighbourhood $\mathcal{V}$ of the origin in $h_{K}^{2, \alpha}\left(\mathbb{S}^{N-1}\right)$ and an analytic operator $\psi_{K}: \mathcal{V} \rightarrow M_{K}^{N}$ defined by

$$
\psi_{K}(r)=\phi_{K}^{-1}(0, r)
$$

By straightforward calculation we see that $\psi_{K}^{\prime}(0)$ is the identity on $\mathcal{V}$. Let $\mathcal{G}_{K}: h_{K}^{2, \alpha}\left(\mathbb{S}^{N-1}\right) \rightarrow$ $h^{1, \alpha}\left(\mathbb{S}^{N-1}\right)$ be the restriction of $\mathcal{F}_{2}$ to $h_{K}^{2, \alpha}\left(\mathbb{S}^{N-1}\right)$. It is easy to see that

$$
\mathcal{G}_{K}^{\prime}(0)=\left.\left(-\frac{1}{\sigma_{N}} \mathcal{N}-\frac{N}{\sigma_{N}} \mathcal{I}\right)\right|_{h_{K}^{2, \alpha}\left(\mathbb{S}^{N-1}\right)} .
$$

LEMma 4.2 The spectrum of $\mathcal{G}_{K}^{\prime}(0)$ consists entirely of eigenvalues and is equal to

$$
\sigma\left(\mathcal{G}_{K}^{\prime}(0)\right)=\sigma\left(\mathcal{F}_{2}^{\prime}(0)\right) \backslash\left\{-\frac{N}{\sigma_{N}},-\frac{N+1}{\sigma_{N}}, \ldots,-\frac{N+K}{\sigma_{N}}\right\} .
$$

Proof. This is proved in [25, Lemma 4.4]. The proof is based on the fact that $\mathcal{F}_{2}^{\prime}(0)$ respects the decomposition

$$
h^{2, \alpha}\left(\mathbb{S}^{N-1}\right)=h_{K}^{2, \alpha}\left(\mathbb{S}^{N-1}\right) \oplus \bigoplus_{k=1}^{K} S_{k}^{N} .
$$

TheOREM 4.3 Let $0<\lambda_{0}<(N+K+1) / \sigma_{N}$. There exists a $\delta>0$ and an $M>0$ such that the problem

$$
\frac{\partial r}{\partial \tau}=\mathcal{F}_{2}(r)
$$

with $r(0)=r_{0} \in M_{K}^{N}$ and $\left\|r_{0}\right\|_{\mathcal{C}^{2, \alpha}\left(\mathbb{S}^{N-1}\right)}<\delta$ has a solution $r \in \mathcal{C}\left([0, \infty), h^{2, \alpha}\left(\mathbb{S}^{N-1}\right)\right) \cap$ $\mathcal{C}^{1}\left([0, \infty), h^{1, \alpha}\left(\mathbb{S}^{N-1}\right)\right)$ satisfying

$$
\|r(\tau)\|_{\mathcal{C}^{2, \alpha}\left(\mathbb{S}^{N-1}\right)} \leqslant M e^{-\lambda_{0} \tau}\left\|r_{0}\right\|_{\mathcal{C}^{2, \alpha}\left(\mathbb{S}^{N-1}\right)} .
$$

Proof. Existence follows from Theorem 3.3 Note that if $r \in M_{K}^{N}$ then

$$
\psi_{K}\left(\mathcal{P}_{K} r\right)=r .
$$

We have

$$
\frac{\partial\left(\mathcal{P}_{K} r\right)}{\partial \tau}=\mathcal{P}_{K}\left(\frac{\partial r}{\partial \tau}\right)=\mathcal{P}_{K}\left(\mathcal{F}_{2}(r)\right)=\left(\mathcal{P}_{K} \circ \mathcal{F}_{2} \circ \psi_{K}\right)\left(\mathcal{P}_{K} r\right)
$$


This is an evolution equation for $\mathcal{P}_{K} r$. Linearising the evolution operator $\mathcal{P}_{K} \circ \mathcal{F}_{2} \circ \psi_{K}$ : $h_{K}^{2, \alpha}\left(\mathbb{S}^{N-1}\right) \cap \mathcal{U} \rightarrow h_{K}^{1, \alpha}\left(\mathbb{S}^{N-1}\right)$ around zero leads to

$$
\left(\mathcal{P}_{K} \circ \mathcal{F}_{2} \circ \psi_{K}\right)^{\prime}(0)=\mathcal{G}_{K}^{\prime}(0) .
$$

$\mathcal{G}_{K}^{\prime}(0)$ is sectorial because $\mathcal{F}_{2}^{\prime}(0)$ is sectorial. By Lemma 4.2 and [19, Theorem 9.1.2] we get

$$
\begin{aligned}
\|r(\tau)\|_{\mathcal{C}^{2, \alpha}\left(\mathbb{S}^{N-1}\right)} & =\left\|\left(\psi_{K} \circ \mathcal{P}_{K}\right) r(\tau)\right\|_{\mathcal{C}^{2, \alpha}\left(\mathbb{S}^{N-1}\right)} \leqslant C\left\|\mathcal{P}_{K} r(\tau)\right\|_{\mathcal{C}^{2, \alpha}\left(\mathbb{S}^{N-1}\right)} \\
& \leqslant C e^{-\lambda_{0} \tau}\left\|\mathcal{P}_{K} r_{0}\right\|_{\mathcal{C}^{2, \alpha}\left(\mathbb{S}^{N-1}\right)} \leqslant C e^{-\lambda_{0} \tau}\left\|r_{0}\right\|_{\mathcal{C}^{2, \alpha}\left(\mathbb{S}^{N-1}\right)}
\end{aligned}
$$

Combining the above with 2.13 we get the following estimate for the nonautonomous problem 2.12:

$$
\|r(t)\|_{\mathcal{C}^{2, \alpha}\left(\mathbb{S}^{N-1}\right)} \leqslant M\left(\frac{N t}{\sigma_{N}}+1\right)^{-\zeta}\left\|r_{0}\right\|_{\mathcal{C}^{2, \alpha}\left(\mathbb{S}^{N-1}\right)}
$$

for $\zeta=\lambda_{0} \sigma_{N} / N$

\section{The spectrum of the linearisation and stability for $N=3$ in the case of Hele-Shaw flow} with surface tension

In this section we apply the principle of linearised stability (see [19]) to the evolution equation 2.15) for the three-dimensional problem with surface tension in order to derive a stability result for the injection case. For the suction case we will derive stability if the suction point is the geometric centre of the initial domain and the quotient of suction speed and $\gamma$ is small enough. We need to study the spectral properties of the operator $\mathcal{F}_{\gamma, \mu}^{\prime}(0): h^{4, \alpha}\left(\mathbb{S}^{2}\right) \rightarrow h^{1, \alpha}\left(\mathbb{S}^{2}\right)$ given by

$$
\mathcal{F}_{\gamma, \mu}^{\prime}(0)[h]=\gamma \mathcal{N}\left(-\mathcal{N}^{2} h-\mathcal{N} h+2 h\right)-\frac{\mu}{4 \pi}(\mathcal{N} h+3 h) .
$$

For each $\gamma>0$ and $\mu \in \mathbb{R} \backslash\{0\}$ introduce

$$
g_{k}=\gamma k\left(-k^{2}-k+2\right)-\frac{\mu}{4 \pi}(k+3) .
$$

The following lemma is a simple consequence of (3.1) and 5.1).

LEMMA 5.1 The point spectrum of $\mathcal{F}_{\gamma, \mu}^{\prime}(0): h^{4, \alpha}\left(\mathbb{S}^{2}\right) \rightarrow h^{1, \alpha}\left(\mathbb{S}^{2}\right)$ is

$$
\pi\left(\mathcal{F}_{\gamma, \mu}^{\prime}(0)\right)=\left\{g_{0}, g_{1}, g_{2}, \ldots\right\}
$$

The eigenspace for the eigenvalue $g_{k}$ is $S_{k}^{3}$. If $\mu>0$ then all eigenvalues of $\mathcal{F}_{\gamma, \mu}^{\prime}(0): h^{4, \alpha}\left(\mathbb{S}^{2}\right) \rightarrow$ $h^{1, \alpha}\left(\mathbb{S}^{2}\right)$ are negative. If $\mu<0$ then the eigenvalues $g_{0}$ and $g_{1}$ are positive. All other eigenvalues are negative if

$$
|\mu| / \gamma=-\mu / \gamma<32 \pi / 5
$$

For two Banach spaces $X$ and $Y$ such that $X \hookrightarrow Y$ we define $\mathcal{H}(X, Y)$ as the collection of operators $A \in \mathcal{L}(X, Y)$ for which $-A$ is the infinitesimal generator of a strongly continuous analytic semigroup. 
Lemma 5.2 We have $-\mathcal{F}_{\gamma, \mu}^{\prime}(0) \in \mathcal{H}\left(h^{4, \alpha}\left(\mathbb{S}^{2}\right), h^{1, \alpha}\left(\mathbb{S}^{2}\right)\right)$.

Proof. See Appendix A.

Lemma 5.3 The spectrum of $\mathcal{F}_{\gamma, \mu}^{\prime}(0): h^{4, \alpha}\left(\mathbb{S}^{2}\right) \rightarrow h^{1, \alpha}\left(\mathbb{S}^{2}\right)$ consists entirely of eigenvalues and $\sigma\left(\mathcal{F}_{\gamma, \mu}^{\prime}(0)\right)=\left\{g_{0}, g_{1}, g_{2}, \ldots\right\}$.

The resolvent $\left(\lambda \mathcal{I}-\mathcal{F}_{\gamma, \mu}^{\prime}(0)\right)^{-1}: h^{1, \alpha}\left(\mathbb{S}^{2}\right) \rightarrow h^{1, \alpha}\left(\mathbb{S}^{2}\right)$ is compact for all $\lambda \notin \sigma\left(\mathcal{F}_{\gamma, \mu}^{\prime}(0)\right)$.

Proof. This follows from Lemma 5.2 and the same reasoning as in the proof of Lemma 3.2

THEOREM 5.4 Let $\mu>0$ and $0<\lambda_{0}<3 \mu /(4 \pi)$. There exists a $\delta>0$ and an $M>0$ such that the problem

$$
\frac{\partial r}{\partial \tau}=\mathcal{F}_{\gamma, \mu}(r)
$$

with $r(0)=r_{0} \in h^{4, \alpha}\left(\mathbb{S}^{2}\right)$ and $\left\|r_{0}\right\|_{\mathcal{C}^{4, \alpha}\left(\mathbb{S}^{2}\right)}<\delta$ has a solution $r \in \mathcal{C}\left([0, \infty), h^{4, \alpha}\left(\mathbb{S}^{2}\right)\right) \cap$ $\mathcal{C}^{1}\left([0, \infty), h^{1, \alpha}\left(\mathbb{S}^{2}\right)\right)$ satisfying

$$
\|r(\tau)\|_{\mathcal{C}^{4, \alpha}\left(\mathbb{S}^{2}\right)} \leqslant M e^{-\lambda_{0} \tau}\left\|r_{0}\right\|_{\mathcal{C}^{4, \alpha}\left(\mathbb{S}^{2}\right)} .
$$

Proof. In Lemma 5.2 we saw that $\mathcal{F}_{\gamma, \mu}^{\prime}(0)$ is sectorial. Note that $-3 \mu /(4 \pi)$ is the largest eigenvalue of $\mathcal{F}_{\gamma, \mu}^{\prime}(0)$. The theorem follows from Lemma 2.3. Lemma 5.3 and [19, Theorem 9.1.2].

If we combine this estimate with (2.13) we get for the nonautonomous problem (2.12) the estimate

$$
\|r(t)\|_{\mathcal{C}^{4, \alpha}\left(\mathbb{S}^{2}\right)} \leqslant M\left(\frac{3 \mu t}{4 \pi}+1\right)^{-\zeta}\left\|r_{0}\right\|_{\mathcal{C}^{4, \alpha}\left(\mathbb{S}^{2}\right)}
$$

for $\zeta=4 \pi \lambda_{0} /(3 \mu)$.

The case $\mu<0$ is more complicated. We need some extra conditions for certain Richardson moments of the initial domain in order to get results similar to Theorem 5.4. Note that $r \in M_{1}^{3}$ if and only if the corresponding domain $\Omega_{r}$ has the volume of the unit ball and its geometric centre is at the origin.

Lemma 5.5 Suppose that $r$ satisfies 2.15. If $r_{0} \in M_{1}^{3}$ then $r(t) \in M_{1}^{3}$ for all $t>0$.

Proof. It is easy to check that if $\Omega_{r(0)}$ has the volume of the unit ball, then so does $\Omega_{r(t)}$ for all $t$. Let $R(t)=s_{N}(t)(1+r(t))-1$ and let $p$ and $v$ be the solutions of $(1.1)-(1.3)$, (1.5) on $\Omega_{R(t)}$. We shall use the following identity for the curvature $\kappa_{R(t)}$ and the Laplace-Beltrami operator $\Delta_{R(t)}$ of $\Gamma_{R(t)}$ :

$$
\kappa_{R(t)}=\left(\Delta_{R(t)} x\right) \cdot n_{R(t)},
$$

where $n_{R(t)}$ is the outer normal on $\Gamma_{R(t)}$ and $x: \Gamma_{R(t)} \rightarrow \mathbb{R}^{3}$ stands for the identity. This is proved in [7, Chapter 2]. Let $x_{j}$ denote the $j$-th component of $x$. By Green's second identity, [1.2], [1.5], (1.6) and the symmetry of the Laplace-Beltrami operator we have

$$
\begin{aligned}
\frac{\mathrm{d}}{\mathrm{d} t} \int_{\Omega_{R(t)}} x_{j} \mathrm{~d} x & =\int_{\Gamma_{R(t)}} x_{j}(v, n) \mathrm{d} x=\int_{\Gamma_{R(t)}}-x_{j} \frac{\partial p}{\partial n} \mathrm{~d} x=-\int_{\Gamma_{R(t)}} p \frac{\partial x_{j}}{\partial n} \mathrm{~d} x \\
& =\gamma \int_{\Gamma_{R(t)}} \kappa_{R(t)} n_{j} \mathrm{~d} x=\gamma \int_{\Gamma_{R(t)}} \Delta_{R(t)} x_{j} \mathrm{~d} x=0 .
\end{aligned}
$$

The lemma follows from this. 
THEOREM 5.6 Let $\mu<0$ be such that 5.2 holds and let $0<\lambda_{0}<5 \mu /(4 \pi)+8 \gamma$. There exists a $\delta>0$ and an $M>0$ such that the problem

$$
\frac{\partial r}{\partial \tau}=\mathcal{F}_{\gamma, \mu}(r)
$$

with $r(0)=r_{0} \in h^{4, \alpha}\left(\mathbb{S}^{2}\right) \cap M_{1}^{3}$ and $\left\|r_{0}\right\|_{\mathcal{C}^{4, \alpha}\left(\mathbb{S}^{2}\right)}<\delta$ has a solution $r \in \mathcal{C}\left([0, \infty), h^{4, \alpha}\left(\mathbb{S}^{2}\right)\right) \cap$ $\mathcal{C}^{1}\left([0, \infty), h^{1, \alpha}\left(\mathbb{S}^{2}\right)\right)$ satisfying

$$
\|r(\tau)\|_{\mathcal{C}^{4, \alpha}\left(\mathbb{S}^{2}\right)} \leqslant M e^{-\lambda_{0} \tau}\left\|r_{0}\right\|_{\mathcal{C}^{4, \alpha}\left(\mathbb{S}^{2}\right)} .
$$

Proof. Introduce

$$
\mathcal{G}_{1, \gamma, \mu}=\left.\mathcal{F}_{\gamma, \mu}\right|_{h_{1}^{4, \alpha}\left(\mathbb{S}^{2}\right)}
$$

with $h_{1}^{4, \alpha}\left(\mathbb{S}^{2}\right)$ as defined in Section 4 . We get

$$
\mathcal{G}_{1, \gamma, \mu}^{\prime}(0)=\left.\mathcal{F}_{\gamma, \mu}^{\prime}(0)\right|_{h_{1}^{4, \alpha}\left(\mathbb{S}^{2}\right)} .
$$

Because $\mathcal{F}_{\gamma, \mu}^{\prime}(0)$ is invariant with respect to the decomposition $h^{k, \alpha}\left(\mathbb{S}^{2}\right)=h_{1}^{k, \alpha}\left(\mathbb{S}^{2}\right) \oplus S_{0}^{3} \oplus S_{1}^{3}$, we find

$$
\sigma\left(\mathcal{G}_{1, \gamma, \mu}^{\prime}(0)\right)=\left\{g_{2}, g_{3}, g_{4}, \ldots\right\}
$$

The operator $\mathcal{G}_{1, \gamma, \mu}^{\prime}(0)$ is sectorial because $\mathcal{F}_{\gamma, \mu}^{\prime}(0)$ is sectorial (see Lemma 5.2). Let $\phi_{1}$ : $h^{4, \alpha}\left(\mathbb{S}^{2}\right) \rightarrow \mathbb{R} \times \mathbb{R}^{3} \times h_{1}^{4, \alpha}\left(\mathbb{S}^{2}\right)$ be defined as in Section 4. We showed that there exists a neighbourhood $\mathcal{V} \subseteq h_{1}^{4, \alpha}\left(\mathbb{S}^{2}\right)$ of zero such that $\{0\} \times\{0\} \times \mathcal{V} \subseteq \phi_{1}(\mathcal{U})$ and an analytic mapping $\psi_{1}: \mathcal{V} \rightarrow M_{1}^{3}$ given by

$$
\psi_{1}(\tilde{r})=\phi^{-1}(0,0, \tilde{r}) .
$$

Assume for the moment that $r$ is a solution to 5.4 and $r(t) \in M_{1}^{3} \cap \mathcal{U}$. Then $\tilde{r}=\mathcal{P}_{1} r$ satisfies

$$
\frac{\partial \tilde{r}}{\partial \tau}=\left(\mathcal{P}_{1} \circ \mathcal{F}_{\gamma, \mu} \circ \psi_{1}\right)(\tilde{r}) \text {. }
$$

We will discuss the solvability of (5.5) first. Analogously to Section 4, we find that the linearisation around zero of the evolution operator on the right-hand side is

$$
\left(\mathcal{P}_{1} \circ \mathcal{F}_{\gamma, \mu} \circ \psi_{1}\right)^{\prime}(0)=\mathcal{G}_{1, \gamma, \mu}^{\prime}(0) \text {. }
$$

From [19, Theorem 9.1.2], we get a $\delta>0$ such that if $\tilde{r}_{0}=\mathcal{P}_{1} r_{0} \in h_{1}^{4, \alpha}\left(\mathbb{S}^{2}\right)$ with $\left\|\tilde{r}_{0}\right\|_{\mathcal{C}^{4, \alpha}\left(\mathbb{S}^{2}\right)}<\delta$, then the problem 5.5 with $\tilde{r}(0)=\tilde{r}_{0}$ has a unique solution $\tilde{r} \in \mathcal{C}\left([0, \infty), h_{1}^{4, \alpha}\left(\mathbb{S}^{2}\right)\right) \cap$ $\mathcal{C}^{1}\left([0, \infty), h_{1}^{1, \alpha}\left(\mathbb{S}^{2}\right)\right)$. Furthermore, there exists an $M^{\prime}>0$ independent of $\tilde{r}_{0}$ such that

$$
\|\tilde{r}(\tau)\|_{\mathcal{C}^{4, \alpha}\left(\mathbb{S}^{2}\right)} \leqslant M^{\prime} e^{-\lambda_{0} \tau}\left\|\tilde{r}_{0}\right\|_{\mathcal{C}^{4, \alpha}\left(\mathbb{S}^{2}\right)} .
$$

Set

$$
r=\psi_{1}(\tilde{r}) .
$$


Then

$$
\frac{\partial r}{\partial \tau}=\psi_{1}^{\prime}(\tilde{r})\left[\frac{\partial \tilde{r}}{\partial \tau}\right]=\psi_{1}^{\prime}\left(\mathcal{P}_{1} r\right)\left[\mathcal{P}_{1} \mathcal{F}_{\gamma, \mu}(r)\right] .
$$

Because $\psi_{1}\left(\mathcal{P}_{1} r\right)=r$ for all $r \in M_{1}^{3} \cap \mathcal{U}$, we have

$$
\psi_{1}^{\prime}\left(\mathcal{P}_{1} r\right)\left[\mathcal{P}_{1} h\right]=h
$$

for all $h \in T_{r} M_{1}^{3}$. By Lemma 5.5 we have $\mathcal{F}_{\gamma, \mu}(r) \in T_{r} M_{1}^{3}$ and therefore

$$
\frac{\partial r}{\partial \tau}=\mathcal{F}_{\gamma, \mu}(r)
$$

There exists a $\delta>0$ and an $M>0$ such that for $r_{0} \in M_{1}^{3}$ with $\left\|r_{0}\right\|_{\mathcal{C}^{4, \alpha}\left(\mathbb{S}^{2}\right)}<\delta$ we have

$$
\|r(\tau)\|_{\mathcal{C}^{4, \alpha}\left(\mathbb{S}^{2}\right)} \leqslant M e^{-\lambda_{0} \tau}\left\|r_{0}\right\|_{\mathcal{C}^{4, \alpha}\left(\mathbb{S}^{2}\right)} .
$$

This estimate follows exactly in the same way as in the proof of Theorem 4.3 This proves the theorem.

If we combine this estimate with (2.13) we get for the nonautonomous problem (2.12) the estimate

$$
\|r(t)\|_{\mathcal{C}^{4, \alpha}\left(\mathbb{S}^{2}\right)} \leqslant M\left(\frac{3 \mu t}{4 \pi}+1\right)^{\zeta}\left\|r_{0}\right\|_{\mathcal{C}^{4, \alpha}\left(\mathbb{S}^{2}\right)}
$$

for $\zeta=-4 \pi \lambda_{0} /(3 \mu)$ and $t \in\left[0, T_{\mu}\right)$

\section{Stability for perturbations of the suction point}

If the suction point is not at the geometric centre of the initial domain we cannot derive a result like Theorem 5.6. As shown in [23], the solution either becomes unbounded or breaks down before all liquid is sucked out. In this section we show that for the problem with surface tension an arbitrarily large portion of the liquid can be removed if $r_{0}$ is sufficiently small. We do not need to restrict ourselves to the case $r_{0} \in M_{1}^{3}$.

LemMA 6.1 Let $\mu<0, \alpha_{1} \in(0, \alpha), \beta \in(\alpha, 1)$ and assume that 5.2 holds. There exists a neighbourhood $\mathcal{U}$ of 0 in $h^{3, \beta}\left(\mathbb{S}^{2}\right)$ such that the problem

$$
\frac{\partial r}{\partial \tau}=\mathcal{F}_{\gamma, \mu}(r)
$$

has for each $r(0)=r_{0} \in \mathcal{U} \cap h^{4, \alpha_{1}}\left(\mathbb{S}^{2}\right)$ a unique maximal solution

$$
r \in \mathcal{C}\left(\left[0, T^{+}\left(r_{0}\right)\right), h^{4, \alpha_{1}}\left(\mathbb{S}^{2}\right)\right) \cap \mathcal{C}^{0, \eta}\left(\left[0, T^{+}\left(r_{0}\right)\right), h^{1, \alpha}\left(\mathbb{S}^{2}\right)\right),
$$

where $\eta=1-\left(\alpha-\alpha_{1}\right) / 3$. The mapping $\left(r_{0}, \tau\right) \mapsto r(\tau)$ is a semiflow on $\mathcal{U} \cap h^{4, \alpha_{1}}\left(\mathbb{S}^{2}\right)$.

Proof. According to [12, Lemma 3.1], there exists a neighbourhood $\hat{\mathcal{U}}$ of 0 in $h^{2, \beta}\left(\mathbb{S}^{2}\right)$ and

$$
\kappa_{1} \in \mathcal{C}^{\omega}\left(\hat{\mathcal{U}}, \mathcal{L}\left(h^{3, \alpha}\left(\mathbb{S}^{2}\right), h^{1, \alpha}\left(\mathbb{S}^{2}\right)\right)\right), \quad \kappa_{2} \in \mathcal{C}^{\omega}\left(\hat{\mathcal{U}}, h^{1, \beta}\left(\mathbb{S}^{2}\right)\right)
$$


such that

$$
\kappa(r)=\kappa_{1}(r) r+\kappa_{2}(r) .
$$

From (5.3), we see that $\kappa_{1}$ is a quasilinear differential operator of second order and $\kappa_{2}$ is of first order. Therefore there exists a small neighbourhood $\mathcal{U} \subset \hat{\mathcal{U}}$ of 0 in $h^{3, \beta}\left(\mathbb{S}^{2}\right)$ such that

$$
\kappa_{1} \in \mathcal{C}^{\omega}\left(\mathcal{U}, \mathcal{L}\left(h^{4, \alpha}\left(\mathbb{S}^{2}\right), h^{2, \alpha}\left(\mathbb{S}^{2}\right)\right)\right)
$$

and

$$
\kappa_{2} \in \mathcal{C}^{\omega}\left(\mathcal{U}, h^{2, \beta}\left(\mathbb{S}^{2}\right)\right) .
$$

Combining this with Lemma 2.3 we can choose $\mathcal{U}$ such that

$$
r \mapsto \mathcal{E}(r) \kappa_{1}(r) \in \mathcal{C}^{\omega}\left(\mathcal{U}, \mathcal{L}\left(h^{4, \alpha}\left(\mathbb{S}^{2}\right), h^{1, \alpha}\left(\mathbb{S}^{2}\right)\right)\right) .
$$

By [12, Remark 3.3] we have

$$
\mathcal{E}(0) \kappa_{1}(0)=\mathcal{N}^{3}+p(\mathcal{N}),
$$

where $p$ is a polynomial of degree 2 and therefore from Lemma 5.2 we get

$$
\mathcal{E}(0) \kappa_{1}(0) \in \mathcal{H}\left(h^{4, \alpha}\left(\mathbb{S}^{2}\right), h^{1, \alpha}\left(\mathbb{S}^{2}\right)\right),
$$

because $\mathcal{E}(0) \kappa_{1}(0)$ is in highest order equal to $-\mathcal{F}_{\gamma, \mu}^{\prime}(0)$. By [3, Theorem I.1.3.1], the set $\mathcal{H}\left(h^{4, \alpha}\left(\mathbb{S}^{2}\right), h^{1, \alpha}\left(\mathbb{S}^{2}\right)\right)$ is open in $\mathcal{L}\left(h^{4, \alpha}\left(\mathbb{S}^{2}\right), h^{1, \alpha}\left(\mathbb{S}^{2}\right)\right)$. This implies that we can choose $\mathcal{U}$ such that

$$
r \mapsto \gamma \mathcal{E}(r) \kappa_{1}(r) \in \mathcal{C}^{\omega}\left(\mathcal{U}, \mathcal{H}\left(h^{4, \alpha}\left(\mathbb{S}^{2}\right), h^{1, \alpha}\left(\mathbb{S}^{2}\right)\right)\right) .
$$

By Lemma 2.3, we can choose $\mathcal{U}$ such that

$$
r \mapsto \gamma \mathcal{E}(r) \kappa_{2}(r)+\mu \mathcal{E}(r) \phi(r)+\mu l(r) \in \mathcal{C}^{\omega}\left(\mathcal{U}, h^{1, \beta}\left(\mathbb{S}^{2}\right)\right) .
$$

Because little Hölder spaces satisfy

$$
\left(h^{4, \alpha}\left(\mathbb{S}^{2}\right), h^{1, \alpha}\left(\mathbb{S}^{2}\right)\right)_{1-\left(\alpha-\alpha_{1}\right) / 3, \infty}^{0}=h^{4, \alpha_{1}}\left(\mathbb{S}^{2}\right),
$$

the result follows from 6.1, 6.2) and [2, Theorem 12.1].

Theorem 6.2 Let $T>0, \eta \in(0,1)$ and $\mu<0$ be such that 5.2$)$ holds. Define

$$
\alpha_{1}=\alpha+3(\eta-1) \text {. }
$$

There exists a $\delta>0$ such that the problem

$$
\frac{\partial r}{\partial \tau}=\mathcal{F}_{\gamma, \mu}(r)
$$

with $r(0)=r_{0} \in h^{4, \alpha_{1}}\left(\mathbb{S}^{2}\right)$ and $\left\|r_{0}\right\|_{\mathcal{C}^{4, \alpha_{1}}\left(\mathbb{S}^{2}\right)}<\delta$ has a solution $r \in \mathcal{C}\left([0, T), h^{4, \alpha_{1}}\left(\mathbb{S}^{2}\right)\right) \cap$ $\mathcal{C}^{0, \eta}\left([0, T), h^{1, \alpha}\left(\mathbb{S}^{2}\right)\right)$.

Proof. From the semiflow property proved in Lemma 6.1 we see that the set

$$
V=\left\{\left(r_{0}, \tau\right) \in \mathcal{U} \times(0, \infty): \tau<T^{+}\left(r_{0}\right)\right\}
$$

is open in $h^{4, \alpha_{1}}\left(\tilde{\mathbb{U}}^{2}\right) \times(0, \infty)$. Since $T^{+}(0)=\infty$, the point $(0, T)$ is in $V$. Therefore there exists a neighbourhood $\tilde{\mathcal{U}}$ of zero in $h^{4, \alpha_{1}}\left(\mathbb{S}^{2}\right)$ such that $T^{+}\left(r_{0}\right) \geqslant T$ for all $r_{0} \in \tilde{\mathcal{U}}$.

Note that by the same reasoning one can show that for any $T>0$ and $\hat{r} \in \mathcal{U} \cap h^{4, \alpha_{1}}\left(\mathbb{S}^{2}\right)$ with $T^{+}(\hat{r})=\infty$ there exists a neighbourhood $\tilde{\mathcal{U}}$ of $\hat{r}$ in $h^{4, \alpha_{1}}\left(\mathbb{S}^{2}\right)$ such that if $r_{0} \in \tilde{\mathcal{U}}$ then $T^{+}\left(r_{0}\right) \geqslant T$. 


\section{Appendix A. Proof of Lemma 5.2}

The structure of this proof is as follows. We relate $\mathcal{N}^{3}$ to a Fourier multiplier operator $\hat{\mathcal{N}}_{0}^{3}$ on $\mathbb{R}^{2}$. The operator $-\hat{\mathcal{N}}_{0}^{3}$ generates an analytic semigroup. Using techniques from [13], [12] and [9] together with additional perturbation arguments we see that $\mathcal{N}^{3} \in \mathcal{H}\left(h^{4, \alpha}\left(\mathbb{S}^{2}\right), h^{1, \alpha}\left(\mathbb{S}^{2}\right)\right)$. Since $-\mathcal{F}_{\gamma, \mu}^{\prime}(0)$ is in highest order equal to $\mathcal{N}^{3}$ the lemma follows.

1. Let $\left(\mathcal{T}_{i}, \Xi_{i}\right)_{i=1}^{M}$ be an atlas of $\mathbb{S}^{2}$, with $\Xi_{i}\left(\mathcal{T}_{i}\right)=\tilde{U}_{i}$ and $0 \in \tilde{U}_{i}$. Define

$$
U_{i}=\tilde{U}_{i} \times(0, \varrho)
$$

for some $\varrho<1$,

$$
\mathcal{W}_{i}=\left\{x \in \overline{\mathbb{B}^{3}}: 1-\varrho<|x|<1, x /|x| \in \mathcal{T}_{i}\right\}
$$

and $\mathcal{X}_{i}: \mathcal{W}_{i} \rightarrow U_{i}$ by

$$
\mathcal{X}_{i}(x)=\left(\Xi_{i}(x /|x|), 1-|x|\right) .
$$

Let $\hat{\mathcal{A}}_{i}: h^{2, \alpha}\left(U_{i}\right) \rightarrow h^{0, \alpha}\left(U_{i}\right)$ and $\hat{\mathcal{Q}}_{i}: h^{2, \alpha}\left(U_{i}\right) \rightarrow h^{1, \alpha}\left(\tilde{U}_{i}\right)$ be

$$
\hat{\mathcal{A}}_{i} p=\Delta\left(p \circ \mathcal{X}_{i}\right) \circ \mathcal{X}_{i}^{-1}, \quad \hat{\mathcal{Q}}_{i} p=\frac{\partial}{\partial n}\left(p \circ \mathcal{X}_{i}\right) \circ \mathcal{X}_{i}^{-1}=-\frac{\partial p}{\partial x_{3}},
$$

where $n$ is the normal on $\mathbb{S}^{2}$ and

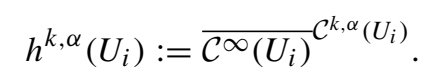

From now on we restrict our attention to one chart and omit the index $i$ in $\tilde{U}_{i}, U_{i}, \hat{\mathcal{A}}_{i}$ and $\hat{\mathcal{Q}}_{i}$. There exist functions $\hat{a}_{j k}, \hat{a}_{j} \in \mathcal{C}^{\infty}(U)$ such that

$$
\hat{\mathcal{A}}=\sum_{j, k=1}^{3} \hat{a}_{j k} \frac{\partial^{2}}{\partial x_{j} \partial x_{k}}-\sum_{j=1}^{3} \hat{a}_{j} \frac{\partial}{\partial x_{j}} .
$$

Define

$$
\hat{\mathcal{A}}_{0}=-1+\sum_{j, k=1}^{3} \hat{a}_{j k}(0) \frac{\partial^{2}}{\partial x_{j} \partial x_{k}} .
$$

Note that $\hat{a}_{33}(0)=1$ and $\hat{a}_{13}(0)=\hat{a}_{23}(0)=\hat{a}_{31}(0)=\hat{a}_{32}(0)=0$. Let $\operatorname{Tr}$ denote the trace operator for functions on the halfspace $\left.\mathbb{R}_{+}^{3}=\left\{x \in \mathbb{R}^{3}: x_{3} \geqslant 0\right)\right\}$. Define $\hat{\mathcal{R}}_{0}: h^{1, \alpha}\left(\mathbb{R}^{2} \times\{0\}\right) \rightarrow h^{1, \alpha}\left(\mathbb{R}_{+}^{3}\right)$ as the solution operator $\hat{\mathcal{R}}_{0} g=u$ of the problem

$$
\begin{cases}-\hat{\mathcal{A}}_{0} u=0 & \text { in } \mathbb{R}_{+}^{3} \\ \operatorname{Tr} u=g & \text { in } \mathbb{R}^{2} \times\{0\}\end{cases}
$$

Define the operator $\hat{\mathcal{N}}_{0}$ by

$$
\hat{\mathcal{N}}_{0}=\hat{\mathcal{Q}} \hat{\mathcal{R}}_{0}
$$

From (4.10) in [13] we get

$$
\mathbb{F} \hat{\mathcal{N}}_{0} \mathbb{F}^{-1}=\mathcal{M}_{f(\cdot, 1)},
$$


where $\mathbb{F}$ denotes Fourier transform, $f: \mathbb{R}^{2} \times \mathbb{R} \rightarrow \mathbb{R}$ is defined by

$$
f(x, y)=\sqrt{y^{2}+\sum_{j, k=1}^{2} \hat{a}_{j k}(0) x_{j} x_{k}},
$$

and $\mathcal{M}_{f(\cdot, 1)}$ stands for multiplication with the function $f(\cdot, 1)$. As $(f(x, y))^{3}$ is positively homogeneous and its derivatives are bounded on $|x|^{2}+y^{2}=1, \hat{\mathcal{N}}_{0}^{3} \in \mathcal{H}\left(h^{4, \alpha}\left(\mathbb{R}^{2} \times\{0\}\right), h^{1, \alpha}\left(\mathbb{R}^{2} \times\{0\}\right)\right)$ (see [10, Theorem A.2]). In [12, Corollary 5.2], the same strategy is used for a different operator.

2. The next step is relating $\hat{\mathcal{N}}_{0}^{3}$ to $\mathcal{N}^{3}$ if the chart domains are small. The following statement holds true. For any $\varepsilon>0$ and $\zeta \in(0, \alpha)$ there is a $\varrho>0$, an atlas $\left(\mathcal{T}_{i}, \Xi_{i}\right)_{i=1}^{M}$, a partition of unity $\left(\psi_{i}\right)_{i=1}^{M}$ subordinate to $\left(\mathcal{W}_{i}\right)_{i=1}^{M}$, and a $C>0$ such that for $l \in\{1,2,3\}$ and $\hat{\mathcal{N}}_{0}$ constructed from the atlas as described above we have, for all $p \in h^{l+1, \alpha}\left(\mathbb{S}^{2}\right)$,

$$
\left\|\mathcal{X}_{*}(\psi \mathcal{N} p)-\hat{\mathcal{N}}_{0} \mathcal{X}_{*}(\psi p)\right\|_{\mathcal{C}^{l, \alpha}\left(\mathbb{R}^{2}\right)} \leqslant \varepsilon\left\|\mathcal{X}_{*}(\psi p)\right\|_{\mathcal{C}^{l+1, \alpha}\left(\mathbb{R}^{2}\right)}+C\|p\|_{\mathcal{C}^{l+1, \zeta}\left(\mathbb{S}^{2}\right)} .
$$

To see this, we argue as in the proof of Theorem B.4 in [9] and choose $\varrho$ sufficiently small, depending on $\varepsilon$. Here and below we identify $\mathcal{C}^{l, \alpha}\left(\mathbb{R}^{2}\right)$ and $\mathcal{C}^{l, \alpha}\left(\mathbb{R}^{2} \times\{0\}\right)$. Functions $\mathcal{X}_{*}(\psi p)$ can be extended to the entire $\mathbb{R}^{2}$ because of the smoothness of the partition of unity. Recall that

$$
\mathcal{X}_{*} f:=f \circ \mathcal{X}^{-1} \text {. }
$$

We want to show that for fixed $\zeta \in(0, \alpha)$ and $\varepsilon>0$, we can derive from A.2 that there is a $C>0$ and an atlas such that for all $p \in h^{4, \alpha}\left(\mathbb{S}^{2}\right)$,

$$
\left\|\mathcal{X}_{*}\left(\psi \mathcal{N}^{3} p\right)-\hat{\mathcal{N}}_{0}^{3} \mathcal{X}_{*}(\psi p)\right\|_{\mathcal{C}^{1, \alpha}\left(\mathbb{R}^{2}\right)} \leqslant \varepsilon\left\|\mathcal{X}_{*}(\psi p)\right\|_{\mathcal{C}^{4, \alpha}\left(\mathbb{R}^{2}\right)}+C\|p\|_{\mathcal{C}^{4, \zeta}\left(\mathbb{S}^{2}\right)}
$$

First we show that there exists a constant $C^{\prime}$ independent of $p$ such that

$$
\left\|\mathcal{X}_{*}(\psi \mathcal{N} p)\right\|_{\mathcal{C}^{3, \alpha}\left(\mathbb{R}^{2}\right)} \leqslant C^{\prime}\left(\left\|\mathcal{X}_{*}(\psi p)\right\|_{\mathcal{C}^{4, \alpha}\left(\mathbb{R}^{2}\right)}+\|p\|_{\mathcal{C}^{4, \zeta}\left(\mathbb{S}^{2}\right)}\right)
$$

and

$$
\left\|\mathcal{X}_{*}\left(\psi \mathcal{N}^{2} p\right)\right\|_{\mathcal{C}^{2, \alpha}\left(\mathbb{R}^{2}\right)} \leqslant C^{\prime}\left(\left\|\mathcal{X}_{*}(\psi p)\right\|_{\mathcal{C}^{4, \alpha}\left(\mathbb{R}^{2}\right)}+\|p\|_{\mathcal{C}^{4, \zeta}\left(\mathbb{S}^{2}\right)}\right) .
$$

Estimate (A.4) follows if we apply (A.2) with $\varepsilon=1$ and $l=3$ together with the boundedness of $\hat{\mathcal{N}}_{0}: h^{k+1, \alpha}\left(\mathbb{R}^{2} \times\{0\}\right) \rightarrow h^{k, \alpha}\left(\mathbb{R}^{2} \times\{0\}\right)$. Estimate A.5 follows in a similar way from A.2, A.4 and the boundedness of $\mathcal{N}: h^{k+1, \alpha}\left(\mathbb{S}^{2}\right) \rightarrow h^{k, \alpha}\left(\mathbb{S}^{2}\right)$. Let $\varepsilon>0$. Let $\eta>0$ be a small number to be chosen later. We have

$$
\begin{aligned}
\left\|\mathcal{X}_{*}\left(\psi \mathcal{N}^{3} p\right)-\hat{\mathcal{N}}_{0}^{3} \mathcal{X}_{*}(\psi p)\right\|_{\mathcal{C}^{1, \alpha}\left(\mathbb{R}^{2}\right) \leqslant} & \left\|\mathcal{X}_{*}\left(\psi \mathcal{N}^{3} p\right)-\hat{\mathcal{N}}_{0} \mathcal{X}_{*}\left(\psi \mathcal{N}^{2} p\right)\right\|_{\mathcal{C}^{1, \alpha}\left(\mathbb{R}^{2}\right)} \\
& +\left\|\hat{\mathcal{N}}_{0} \mathcal{X}_{*}\left(\psi \mathcal{N}^{2} p\right)-\hat{\mathcal{N}}_{0}^{2} \mathcal{X}_{*}(\psi \mathcal{N} p)\right\|_{\mathcal{C}^{1, \alpha}\left(\mathbb{R}^{2}\right)} \\
& +\left\|\hat{\mathcal{N}}_{0}^{2} \mathcal{X}_{*}(\psi \mathcal{N} p)-\hat{\mathcal{N}}_{0}^{3} \mathcal{X}_{*}(\psi p)\right\|_{\mathcal{C}^{1, \alpha}\left(\mathbb{R}^{2}\right)}
\end{aligned}
$$

The three terms on the right can be estimated separately. We denote by $C_{\eta}$ constants depending on $\eta$ while $C$ denotes constants independent of $\eta$. Applying A.2 to $\mathcal{N}^{2} p$ with $l=1$ and A.5 we get for the first term the estimate

$$
\begin{aligned}
&\left\|\mathcal{X}_{*}\left(\psi \mathcal{N}^{3} p\right)-\hat{\mathcal{N}}_{0} \mathcal{X}_{*}\left(\psi \mathcal{N}^{2} p\right)\right\|_{\mathcal{C}^{1, \alpha}\left(\mathbb{R}^{2}\right)} \leqslant \eta\left\|\mathcal{X}_{*}\left(\psi \mathcal{N}^{2} p\right)\right\|_{\mathcal{C}^{2, \alpha}\left(\mathbb{R}^{2}\right)}+C_{\eta}\left\|\mathcal{N}^{2} p\right\|_{\mathcal{C}^{2, \zeta}\left(\mathbb{S}^{2}\right)} \\
& \leqslant \eta C\left\|\mathcal{X}_{*}(\psi p)\right\|_{\mathcal{C}^{4, \alpha}\left(\mathbb{R}^{2}\right)}+C_{\eta}\|p\|_{\mathcal{C}^{4, \zeta}\left(\mathbb{S}^{2}\right)} .
\end{aligned}
$$


The other terms can be estimated in a similar way. Finally,

$$
\left\|\mathcal{X}_{*}\left(\psi \mathcal{N}^{3} p\right)-\hat{\mathcal{N}}_{0}^{3} \mathcal{X}_{*}(\psi p)\right\|_{\mathcal{C}^{1, \alpha}\left(\mathbb{R}^{2}\right)} \leqslant \eta C\left\|\mathcal{X}_{*}(\psi p)\right\|_{\mathcal{C}^{4, \alpha}\left(\mathbb{R}^{2}\right)}+C_{\eta}\|p\|_{\mathcal{C}^{4, \zeta}\left(\mathbb{S}^{2}\right)} .
$$

We take $\eta=\varepsilon / C$ and get the desired result $\mathrm{A.3}$.

3. Now we prove that for all $\lambda>0$,

$$
\lambda \mathcal{I}+\mathcal{N}^{3}: h^{4, \alpha}\left(\mathbb{S}^{2}\right) \rightarrow h^{1, \alpha}\left(\mathbb{S}^{2}\right)
$$

is an isomorphism. Note that

$$
\lambda \mathcal{I}+\mathcal{N}^{3}=(\sqrt[3]{\lambda} \mathcal{I}+\mathcal{N})\left(\sqrt[3]{\lambda} e^{2 \pi i / 3} \mathcal{I}+\mathcal{N}\right)\left(\sqrt[3]{\lambda} e^{-2 \pi i / 3} \mathcal{I}+\mathcal{N}\right) .
$$

We have surjectivity of $\mu \mathcal{I}+\mathcal{N}: h^{k+1, \alpha}\left(\mathbb{S}^{2}\right) \rightarrow h^{k, \alpha}\left(\mathbb{S}^{2}\right)$ for $\mu \in \mathbb{C} \backslash-\mathbb{N}_{0}$ and for all $k \in \mathbb{N}$. Surjectivity of $\lambda \mathcal{I}+\mathcal{N}^{3}: h^{4, \alpha}\left(\mathbb{S}^{2}\right) \rightarrow h^{1, \alpha}\left(\mathbb{S}^{2}\right)$ follows if we apply this result for $k=1,2,3$ and $\mu=\sqrt[3]{\lambda}, \sqrt[3]{\lambda} e^{2 \pi i / 3}, \sqrt[3]{\lambda} e^{-2 \pi i / 3}$.

4. There exist $C>0$ and $\lambda_{*}>0$ such that for all $r \in h^{4, \alpha}\left(\mathbb{S}^{2}\right)$ and $\lambda \in \mathbb{C}$ with $\operatorname{Re} \lambda \geqslant \lambda_{*}$ we have

$$
|\lambda|\|r\|_{h^{1, \alpha}\left(\mathbb{S}^{2}\right)}+\|r\|_{h^{4, \alpha}\left(\mathbb{S}^{2}\right)} \leqslant C\left\|\left(\lambda \mathcal{I}+\mathcal{N}^{3}\right) r\right\|_{h^{1, \alpha}\left(\mathbb{S}^{2}\right)} .
$$

This can be obtained from (A.3) via exactly the same procedure that is used in [9. proof of Theorem B.4]. The estimate A.77 and the fact that

$$
\lambda_{*} \mathcal{I}+\mathcal{N}^{3}: h^{4, \alpha}\left(\mathbb{S}^{2}\right) \rightarrow h^{1, \alpha}\left(\mathbb{S}^{2}\right)
$$

is an isomorphism imply that $\mathcal{N}^{3} \in \mathcal{H}\left(h^{4, \alpha}\left(\mathbb{S}^{2}\right), h^{1, \alpha}\left(\mathbb{S}^{2}\right)\right)$ (see [3, Remark I.1.2.1(a)]).

\section{Acknowledgements}

This research has been supported by NWO grant 613.000.433. The author would like to thank Georg Prokert for his help.

\section{REFERENCES}

1. Alt, H. W. Lineare Funktionalanalysis. Springer, Berlin (1999). Zbl 0577.46001

2. Amann, H. Nonhomogeneous linear and quasilinear elliptic and parabolic boundary value problems. Function Spaces, Differential Operators and Nonlinear Analysis (Friedrichroda, 1992), Teubner-Texte Math. 133, Teubner, Stuttgart (1993), 9-126. Zbl 0810.35037 $\mid$ MR 1242579

3. Amann, H. Linear and Quasilinear Parabolic Problems. Vol. I. Monogr. Math. 89, Birkhäuser Boston, Boston, MA (1995). Zbl 0819.35001 MR 1345385

4. Begehr, H., \& Gilbert, R. P. Hele-Shaw type flows in $\mathbf{R}^{n}$. Nonlinear Anal. 10 (1986), 65-85. Zbl 0664.76041 MR 0820659

5. Chen, X., \& Friedman, A. A free boundary problem for an elliptic-hyperbolic system: an application to tumor growth. SIAM J. Math. Anal. 35 (2003), 974-986. Zbl 1054.35144 MR 2049029

6. Constantin, P., \& Pugh, M. Global solutions for small data to the Hele-Shaw problem. Nonlinearity 6 (1993), 393-415. Zbl 0808.35104 MR 1223740 
7. Dierkes, U., Hildebrandt, S., Küster, A., \& Wohlrab, O. Minimal Surfaces. I. Grundlehren Math. Wiss. 295, Springer, Berlin (1992). Zbl 0777.53012 MR 1215267

8. Elliott, C. M., \& Ockendon, J. R. Weak and Variational Methods for Moving Boundary Problems. Res. Notes Math. 59, Pitman, Boston, MA (1982). Zbl 0476.35080 MR 0650455

9. Escher, J., \& Prokert, G. Analyticity of solutions to nonlinear parabolic equations on manifolds and an application to Stokes flow. J. Math. Fluid Mech. 8 (2006), 1-35. Zbl 1102.350481 MR 2205149

10. Escher, J., \& Simonett, G. Maximal regularity for a free boundary problem. Nonlinear Differential Equations Appl. 2 (1995), 463-510. Zbl 0842.35083 MR 1356871

11. Escher, J., \& Simonett, G. Analyticity of the interface in a free boundary problem. Math. Ann. 305 (1996), 439-459. Zbl 0857.76086 MR 1397432

12. Escher, J., \& Simonett, G. Classical solutions for Hele-Shaw models with surface tension. Adv. Differential Equations 2 (1997), 619-642. Zbl 1023.35527 MR 1441859

13. Escher, J., \& Simonett, G. Classical solutions of multidimensional Hele-Shaw models. SIAM J. Math. Anal. 28 (1997), 1028-1047. Zbl 0888.35142 MR 1466667

14. Escher, J., \& Simonett, G. A center manifold analysis for the Mullins-Sekerka model. J. Differential Equations 143 (1998), 267-292. Zbl 0896.35142 MR 1607952

15. Friedman, A., \& Reitich, F. Nonlinear stability of a quasi-static Stefan problem with surface tension: a continuation approach. Ann. Scuola Norm. Sup. Pisa Cl. Sci. (4) 30 (2001), 341-403. Zbl 1072.35208 MR 1895715

16. GUSTAFSSON, B. Applications of variational inequalities to a moving boundary problem for Hele-Shaw flows. SIAM J. Math. Anal. 16 (1985), 279-300. Zbl 0605.76043 MR 0777468

17. Hohlov, YU. E. Some open questions on regularization of ill-posed free boundary problems. FBP News 4 (1994), 11-12.

18. Kato, T. Perturbation Theory for Linear Operators. Classics in Math., Springer, Berlin (1995) (reprint of the 1980 edition). Zbl 0836.47009 MR 1335452

19. Lunardi, A. Analytic Semigroups and Optimal Regularity in Parabolic Problems. Progr. Nonlinear Differential Equations Appl. 16, Birkhäuser, Basel (1995). Zbl 0816.35001 MR 1329547

20. MÜLler, C. Spherical Harmonics. Lecture Notes in Math. 17, Springer, Berlin (1966). Zbl 0138.05101 MR 0199449

21. Prokert, G. Parabolic evolution equations for quasistationary free boundary problems in capillary fluid mechanics. Dissertation, Technische Univ. Eindhoven, Eindhoven (1997). Zbl 0896.35145 MR 1454616

22. Prokert, G. Existence results for Hele-Shaw flow driven by surface tension. Eur. J. Appl. Math. 9 (1998), 195-221. Zbl 0919.35005 MR 1630665

23. Tian, F. R. On the breakdown of Hele-Shaw solutions with nonzero surface tension. J. Nonlinear Sci. 5 (1995), 479-484. Zbl 0862.35093 MR 1361783

24. Vondenhoff, E. Asymptotic behaviour of injection and suction for Hele-Shaw flow in $\mathbb{R}^{3}$ with surface tension near balls. CASA-Report 06-42, Technische Univ. Eindhoven, Eindhoven (2006).

25. Vondenhoff, E. Long-time behaviour of classical Hele-Shaw flows with injection near expanding balls. CASA-Report 06-19, Technische Univ. Eindhoven, Eindhoven (2006). 